\title{
Review Article \\ Impact of Inflammation on Myeloproliferative Neoplasm Symptom Development
}

\author{
Holly L. Geyer, ${ }^{1}$ Amylou C. Dueck, ${ }^{2}$ Robyn M. Scherber, ${ }^{3}$ and Ruben A. Mesa ${ }^{3}$ \\ ${ }^{1}$ Division of Hospital Internal Medicine, Mayo Clinic, Scottsdale, AZ 85054, USA \\ ${ }^{2}$ Department of Statistics, Mayo Clinic, Scottsdale, AZ 85054, USA \\ ${ }^{3}$ Division of Hematology and Medical Oncology, Mayo Clinic, Scottsdale, AZ 85054, USA
}

Correspondence should be addressed to Ruben A. Mesa; mesa.ruben@mayo.edu

Received 23 June 2015; Accepted 9 August 2015

Academic Editor: Sylvie Hermouet

Copyright (C) 2015 Holly L. Geyer et al. This is an open access article distributed under the Creative Commons Attribution License, which permits unrestricted use, distribution, and reproduction in any medium, provided the original work is properly cited.

\begin{abstract}
Myeloproliferative neoplasms (essential thrombocythemia, ET; polycythemia vera, PV; myelofibrosis, MF) are monoclonal malignancies associated with genomic instability, dysregulated signaling pathways, and subsequent overproduction of inflammatory markers. Acknowledged for their debilitating symptom profiles, recent investigations have aimed to determine the identity of these markers, the upstream sources stimulating their development, their prevalence within the MPN population, and the role they play in symptom development. Creation of dedicated Patient Reported Outcome (PRO) tools, in combination with expanded access to cytokine analysis technology, has resulted in a surge of investigations evaluating the potential associations between symptoms and inflammation. Emerging data demonstrates clear relationships between individual MPN symptoms (fatigue, abdominal complaints, microvascular symptoms, and constitutional symptoms) and cytokines, particularly IL-1, IL-6, IL-8, and TNF- $\alpha$. Information is also compiling on the role symptoms paradoxically play in the development of cytokines, as in the case of fatigue-driven sedentary lifestyles. In this paper, we explore the symptoms inherent to the MPN disorders and the potential role inflammation plays in their development.
\end{abstract}

\section{Introduction}

In 1951, Sir William Dameshek postulated the concept of "myeloproliferative disorders" and furthermore ascribed their development to "a hitherto of undiscovered stimulus." The past half-century has since brought light to the cryptic "stimulus" believed to drive these disabling neoplasms. Developing from a host of myelostimulatory mutations, myeloproliferative neoplasms (MPNs) including polycythemia vera (PV), essential thrombocythemia (ET), and myelofibrosis (MF) propagate through an evolving cascade of inflammatory conduits well documented to inflict dramatic symptomatology and impair quality of life. Great gains have been made in our understanding of how these disrupted signaling pathways coalesce into dysregulated synthesis of cytokines, chemokines, and reactive species that ultimately induce symptoms. In this paper, we discuss the role inflammation plays in MPN pathobiology, disease advancement, and symptom development.

\section{Characterization of MPN Symptoms}

The burdensome symptom profile is arguably the most recognizable feature of the MPN disease process and in itself may contribute to reduced life expectancy, as observed in myelofibrosis risk scoring $[1,2]$. Prominent symptoms include fatigue ( $92.7 \%$ ), early satiety (61.9\%), abdominal pain (45.9\%), abdominal discomfort (53.2\%), inactivity (60.5\%), headache $(48.3 \%)$, concentration problems $(61.7 \%)$, dizziness (55.2\%), numbness (61.3\%), insomnia (65.4\%), sad mood (62.7\%), sexuality problems $(57.9 \%)$, cough $(46.4 \%)$, night sweats $(56.4 \%)$, itching $(52.6 \%)$, bone pain $(48.5 \%)$, fever (20.2\%), weight loss $(34.2 \%)$, and impaired quality of life (84.2\%) [3]. The MPN symptom burden has been closely examined for its impact on patient daily living through the MPN LANDMARK survey. This study systematically surveyed 813 MPN patients and discovered that MPN symptoms negatively impacted work hours, number of sick days taken, the need for medical disability and/or early retirement, 
and overall activities of daily living. Patients additionally described feeling anxious and worried about their conditions (MF, 91\%; PV, 78\%; ET, 74\%) which in turn compromised overall quality of life (MF, 81\%; PV, 66\%; ET, 57\%) [4]. Adding to the complexity is the recent revelation that MPN symptoms indeed promote the development of other symptoms. An investigation of the symptom of insomnia revealed that the complaint correlates closely with most other MPN related symptoms and functional domains [5]. A similar study investigating correlations with MPN-related sexuality complaints found that this symptom also correlated with other MPN symptoms (insomnia, depression/sad mood, night sweats, and QOL), as well as emotional, cognitive, and social domains of functioning [6].

It has been well recognized that the prevalence and severity of symptoms differ by MPN subtype. However, more recent studies have demonstrated that significant heterogeneity exists even within MPN subtypes. A prospective evaluation of 1470 MPN patients discovered the presence of five clusters in PV and ET, respectively, and four clusters in MF [7]. Symptom clusters in ET and PV differed by clinical variables including age, language, gender, the presence of laboratory abnormalities, spleen size, history of hemorrhage, and Myeloproliferative Neoplasm Symptom Assessment Form Total Symptom Score (MPN-SAF TSS) value. Notably, neither PV nor ET clusters differed by risk scores suggesting symptomatology likely presents independent of disease stage and risk scoring tools should not be applied as surrogate measurements of disease severity. In MF, clusters differed by a variety of clinical variables as well as risk scores (DIPSS) with increasing degrees of symptomatology correlating with higher risk score categories.

Recent efforts have aimed to analyze the scope and extent of MPN symptoms in a systematic format. The first investigation was completed in 2007 as a self-reported internet survey of 1179 MPN patients [8]. This revealing study showed that fatigue, pruritus, bone pain, fevers, and weight loss led to restricted participation in physical and social functions and furthermore that available treatment regimens including androgens, steroids, hydroxyurea, and erythropoiesisstimulating agents not only failed to improve the symptom burden but paradoxically contributed to its development. This survey served as a benchmark for the development of three MPN-specific PRO tools: MF-SAF, MPN-SAF, and MPN-SAF TSS/MPN-10. From these instruments, we have gathered key information on both the spectrum and severity of MPN symptoms. Below we discuss these tools individually (Table 1).

2.1. MF-SAF. The Myelofibrosis Symptom Assessment Form (MF-SAF) was created in 2009 and served as the first validated MPN Patient Reported Outcome (PRO) tool to be made available for clinical and trial settings [9]. A 20-item instrument, the survey attempted to capture the most common symptoms within myelofibrosis and content included issues related to catabolic/proliferative symptoms, quality of life, fatigue, and splenomegaly-associated issues. Questions were constructed in a "yes," "no," or 0 (absent) to 10 (worst
TABLE 1: MPN symptom assessment forms.

\begin{tabular}{|c|c|c|c|c|}
\hline $\begin{array}{l}\text { PRO } \\
\text { tool }\end{array}$ & Year & $\begin{array}{l}\text { Total number } \\
\text { of questions }\end{array}$ & $\begin{array}{l}\text { Question } \\
\text { composition }\end{array}$ & $\begin{array}{l}\text { Available } \\
\text { languages }\end{array}$ \\
\hline $\begin{array}{l}\text { MF- } \\
\text { SAF } \\
{[9]}\end{array}$ & 2009 & 20 & $\begin{array}{l}\text { Fatigue } \\
\text { Inactivity } \\
\text { Bone pain } \\
\text { Cough } \\
\text { Pruritus } \\
\text { Night sweats } \\
\text { Fever } \\
\text { Weight loss } \\
\text { Abdominal } \\
\text { pain/discomfort } \\
\text { Early satiety } \\
\text { Quality of life }\end{array}$ & English \\
\hline $\begin{array}{l}\text { MPN- } \\
\text { SAF } \\
{[3]}\end{array}$ & 2011 & 27 & $\begin{array}{l}\text { Fatigue } \\
\text { Inactivity } \\
\text { Headache } \\
\text { Dizziness } \\
\text { Concentration } \\
\text { problems } \\
\text { Numbness } \\
\text { Insomnia } \\
\text { Sad mood } \\
\text { Sexuality problems } \\
\text { Bone pain } \\
\text { Cough } \\
\text { Pruritus } \\
\text { Night sweats } \\
\text { Fever } \\
\text { Weight loss } \\
\text { Abdominal } \\
\text { pain/discomfort } \\
\text { Early satiety } \\
\text { Quality of life }\end{array}$ & $\begin{array}{l}\text { English } \\
\text { Italian } \\
\text { German } \\
\text { French } \\
\text { Mandarin } \\
\text { Arabic } \\
\text { Spanish } \\
\text { Dutch } \\
\text { Swedish } \\
\text { Portuguese } \\
\text { Japanese } \\
\text { Hebrew } \\
\text { Czech }\end{array}$ \\
\hline $\begin{array}{l}\text { MPN- } \\
\text { SAF } \\
\text { TSS [14] }\end{array}$ & 2013 & 10 & $\begin{array}{l}\text { Fatigue } \\
\text { Inactivity } \\
\text { Concentration } \\
\text { problems } \\
\text { Bone pain } \\
\text { Pruritus } \\
\text { Night sweats } \\
\text { Fever } \\
\text { Weight loss } \\
\text { Abdominal } \\
\text { discomfort } \\
\text { Early satiety }\end{array}$ & $\begin{array}{l}\text { English } \\
\text { Italian } \\
\text { German } \\
\text { French } \\
\text { Mandarin } \\
\text { Arabic } \\
\text { Spanish } \\
\text { Dutch } \\
\text { Swedish } \\
\text { Portuguese } \\
\text { Japanese } \\
\text { Hebrew } \\
\text { Czech }\end{array}$ \\
\hline
\end{tabular}

MF-SAF: Myelofibrosis Symptom Assessment Form; MPN-SAF: Myeloproliferative Neoplasm Symptom Assessment Form; MPN-SAF TSS: Myelofibrosis Symptom Assessment Form Total Symptom Score; PRO: Patient Reported Outcome; QOL: quality of life.

imaginable) scale. The tool proved useful in the open label phase II trial of the JAK2 inhibitor, ruxolitinib [10].

2.2. MPN-SAF. The Myeloproliferative Neoplasm Symptom Assessment Form (MPN-SAF) was developed two years later in efforts to capture the symptoms within PV and ET as well as MF [3]. This survey included the items present within 
the MF-SAF, along with questions related to microvasculature complications such as headaches, concentration problems, lightheadedness, dizziness, vertigo, numbness/tingling, and sexual dysfunction. This expanded version was structured in a similar format to the MF-SAF and proved beneficial in evaluation of a variety of novel targeted compounds including ruxolitinib, LY2784544, SAR302503, Vorinostat, and pegylated interferon [11-13].

2.3. MPN-SAF TSS (MPN-10). The Myeloproliferative Neoplasm Symptom Assessment Form Total Symptom Score (MPN-SAF TSS; MPN-10) is an abbreviated version of the MPN-SAF containing the 10 most symptomatic and pertinent items [14]. This tool allows for rapid administration in clinical and trial formats and has replaced the MPN-SAF in most settings. The survey has been successfully cross-validated against the EORTC QLQ-C30 and is available in a variety of languages including English, Italian, German, French, Mandarin, Arabic, Spanish, Dutch, Swedish, Portuguese, Japanese, Hebrew, and Czech.

\section{Origins of Inflammation in MPN Patients}

In healthy individuals, the inflammatory cascade is driven by a delicate interplay between cellular responses and neurohormonal stimulatory factors/cytokines. Dysregulation of this system is a hallmark feature of the MPNs. Although the initial inciting event has yet to be clearly elucidated, all MPN disorders arise from genetic defects within pluripotent stem cell populations that accumulate over the disease course. JAK2V617F, a member of the Janus kinase signal transduction pathway, was the first recognized mutation inherent to the MPN population (PV 96\%, ET 50\%, and MF 50\%) [15]. The role the Janus kinase cascade (including JAK2, JAK2, JAK3, and TYK2) plays in the signaling of inflammatory cytokines is well documented and profound. As JAKs are essential to the signaling of surface growth factor receptors and cytokines bereft of intrinsic kinase activity, constitutive activation, as observed in JAK2V617F, induces unregulated signaling of STAT transcription factors with resultant cellular growth and propagation [16]. STAT3, in particular, is linked closely with cancer development via activation of immunomodulatory cytokines (IL-6, IL-10, and IL-17), growth factors (FGF, VEGF), and matrix metalloproteinases [17]. These products further induce positive autofeedback through the JAK/STAT pathway, perpetuating cellular malignant potential. In general, cytokines (interleukins, interferons, and soluble growth factors; definitions in Table 2) are important regulators of cellular processes, particularly those involving immunomodulatory activities, cellular growth angiogenesis, and migration [18]. Cytokine dysregulation is believed to be associated with other mutations observed within MPNs (IDH1/2, TET2) but requires additional investigation [19].

Chronic inflammation has been hypothesized to play a supportive role in oncogenesis given its promotion of genomic instability through DNA mutations and epigenetic changes, prevention of tumor immune surveillance, and encouragement of clonal evolution [17, 20, 21]. MPN
TABLE 2: Cytokine descriptions.

\begin{tabular}{ll}
\hline Acronym & Description \\
\hline B2MICG & Beta-2 microglobulin \\
BMP6 & Bone morphogenetic protein 6 \\
CRP & C-reactive protein \\
FGF & Fibroblast growth-factor \\
GCSF & Growth colony stimulating factor \\
HGF & Hepatocyte growth factor \\
Hs-CRP & High-sensitivity C-reactive protein \\
IFN- $\alpha$ & Interferon-alpha \\
IFN- $\gamma$ & Interferon-gamma \\
IFN- $\gamma$-IP & Interferon-gamma inducible protein \\
IP-10 & Interferon-gamma inducible protein 10 \\
IL-1B & Interleukin-1B \\
IL-1RA & Interleukin-1RA \\
IL-2R & Interleukin-2R \\
IL-6 & Interleukin-6 \\
IL-8 & Interleukin-8 \\
IL-10 & Interleukin-10 \\
IL-12 & Interleukin-12 \\
IL-13 & Interleukin-13 \\
IL-15 & Interleukin-15 \\
IL-17 & Interleukin-17 \\
MIG & Monokine-induced by gamma \\
MIP-1 $\beta$ & Macrophage inflammatory protein-1 $\beta$ \\
NF-KB & Nuclear factor-KB \\
PAI1 & Plasminogen activator inhibitor-1 \\
PTX3 & Pentraxin-3 \\
TIMP1 & Tissue inhibitor of metalloproteinase-1 \\
TNF-1 & Tumor necrosis factor-1 \\
TNF- $\alpha$ & Tumor necrosis-factor- $\alpha$ \\
TNF-RII & \\
VCAM1 & Vumor necrosis-factor-RII \\
EGGF & Vascular adhesion molecule \\
\hline & \\
& Erothelial growth factor \\
\hline
\end{tabular}

cells (leukocytes, platelets) with inherent hypersensitivity to cytokines and or growth factors respond in a proliferative fashion with resultant production of more stimulatory factors. As chronic inflammatory conditions, MPN disorders revolve around a perpetual cycle of DNA damage, cellular remodeling, and subsequent fibrosis [22]. This process has been a topic of great interest, especially as it relates to the heterozygous clinical presentation of MPN patients.

\section{Inflammation and MPN Symptom Development}

The relationship between chronic inflammation and MPN symptom development has also been a topic of recent interest. An evaluation of abnormal cytokine expression within myelofibrosis determined that primary myelofibrosis (PMF) patients had significantly increased levels of IL-1B, IL-1RA, 
IL-2R, IL-6, IL-8, IL-10, IL-12, IL-13, and IL-15 and TNF-1, G-CSF, IFN- $\alpha$, MIP- $1 \alpha$, MIP-1 $\beta$, HGF, INF- $\gamma$-IP, and VEGF in addition to reduced IFN- $\gamma$ levels [23]. IL-2R, IL-12, IL-15, and IP-10 were independently predictive of inferior survival. IL-2R and IL-12 were associated with transfusion needs and HGF, MIG, and IL-1RA were associated with marked splenomegaly.

Evaluation of the association between cytokines and MF symptoms was undertaken in 2013 through an ad hoc analysis of 309 MF patients during the blinded phase of the COMFORT-1 trial evaluating ruxolitinib against placebo [24]. Changing levels of five cytokines was significantly associated with change in the MPN-SAF TSS when controlled for arm, visit-by-arm interaction, age, sex, and body mass index (BMI). Cytokines included VCAM1, LEPTIN, TNFRII, TIMP1, and B2MICG. IL-8 appears to play a uniquely important role within MPNs. As a potent chemokine, it has previously been shown within other malignancies to promote angiogenesis, induce leukocyte chemotaxis/activation, and stimulate cellular reproduction. A recent study determined IL- 8 to be associated with elevated levels of circulating blasts and the presence of constitutional symptoms [23]. In polycythemia vera, patients demonstrate increased levels of IL-1RA, IL-4, IL-5, IL-6, IL-7, IL-8, IL-10, IL-12, IL13 , IFN- $\gamma$, GM-CSF, MCP-1, MIP- $1 \alpha$, MIP-1 $\beta$, HGF, IP-10, MIG, MCP-1, PDGF-BB, TNF- $\alpha$, IFN- $\gamma$, and VEGF $[25,26]$. Conversely, PV patients also demonstrate lower levels of EGF and RANTES. PV patients also had significantly elevated levels of IL-7, GM-CSF, MIP-1 $\alpha$, IP-10, MIG, eotaxin, IFN$\gamma$, and VEGF in comparison to primary MF patients. On multivariate analysis, MIP-1 $\beta$ was shown to be associated with inferior survival. In addition, hemoglobin count correlated with IL-4 and MCP-1, hematocrit count correlated with TNF- $\alpha$ and MCP-1, lymphocyte count correlated with IL- 6 and TNF-1, and JAK2V617F mutation status correlated with TNF-1 and PDGF-BB [26]. An analysis of ET patients determined this population to have elevated levels of IL-1B, IL-4, IL-6, IL-8, IL-10, IL-12, HGF, GM-CSF, IFN- $\gamma$, MCP-1, PDGF-BB, TNF- $\alpha$, and VEGF. Interestingly, IL-4, IL-8, GMCSF, IFN- $\gamma$, MCP-1, PDGF-BB, and VEGF appeared to be significantly higher in ET patients when compared to PV populations and may serve as useful markers to distinguish the two disorders [26]. Also within ET patients, polynuclear cell counts were found to correlate with HGF, IL-6, IL-12, MG-CSF, and VEGF whereas red cell counts correlated with PDGF-BB levels. JAK2V617F positive status also correlated with PDGF-BB and TNF- $\alpha$ [26]. In comparing PV and ET patients with vascular complications versus those without complications, no significant differences in cytokine levels were noted. However, in comparing PV and ET patients with a history of vascular events, ET patients have significantly increased levels of IL-4, IL-8, GM-CSF, IFN- $\gamma$, MCP-1, and VEGF [26].

Interestingly, the specific combination of inflammatory markers appears to be as important as the type of factor present. In MF, the combination of TNF- $\alpha$ and TIMP- 1 has been shown to promote survival of CD34+ stem cells whereas the combination of ATP and TNF- $\alpha$ has been shown to reduce proliferation [27]. Specific inflammatory markers are also associated with disease severity and complications. For example, pentraxin and CRP are well established to play a role in thrombosis and atherogenesis. These biomarkers have been associated with the development of major thrombotic events in PV and ET [28]. A recent study also identified low levels of IL-12 in MPN patients with vascular complications [26]. Altered levels of PDGF, FGF, and VEGFb have also been noted in stromal cells of patients with PV, ET, and PMF suggesting proinflammatory cytokines promote bone marrow fibrosis which is well established to contribute to anemia and subsequently fatigue $[29,30]$. Cytokines (BMP-1, BMP$\mathrm{R} 2, \mathrm{BMP}-6$, and BMP-7) may have a role in promoting the advancement of MPNs from early to later stages [31]. Of special interest, the presence of specific gene mutations impacts the type and degree of cytokine expression. For instance, JAK2V617F positive patients have significantly higher levels of IL1B, IL-8, IL-17A, and IFN versus triple-negative (JAK2, $M P L$ negative) patients [32]. Much has yet to be learned about the role cytokines play in MPN symptom development. The growing availability of cost-sensitive cytokine profile testing has offered us what can best be recognized as preliminary data on this complex topic. Below we discuss the available literature on specific MPN symptoms and their relationships to inflammation (Table 3).

4.1. Fatigue. Fatigue is a common complaint among cancer patients, present within $30-60 \%$ of the cancer population [33]. The symptom is particularly prominent within MPNs (PV 85\%, ET 72\%, and MF 84\%), representing the most common symptom voiced regardless of subtype. MPN-fatigue has been shown to correlate closely with functional capacities. In a novel evaluation of MPN patient functionality, participants were found to perform an average of 25.1 metabolic equivalents (METS), akin to scores observed in Parkinson disease patients and dramatically lower than healthy controls (45.8 METS) [9].

Cytokines have been documented to induce fatigue in both malignant and nonmalignant states. A recent study identified positive associations between TNF- $\alpha$ and postchemotherapy fatigue in women with breast cancer [34]. Anemia is a recognized contributor to this symptom and recent studies have demonstrated that cytokines play a critical role in the development and perpetuation of this comorbidity. IL-1, IL-6, and TNF were recently shown to promote deregulation of erythropoietin with resultant anemia in acute myelogenous leukemia (AML) and myelodysplastic syndromes (MDS) $[35,36]$. Cytokine-induced hypocortisolism is also a potential source of fatigue. Cytokines have been shown to induce dysregulation of the HPA axis and promote a blunted stress response due to subtherapeutic cortisol production [37]. In cancer, fatigue has been closely associated with depressed mood and increased levels of IL-6, a cytokine observed within MPNs [38, 39]. A survey of 1788 MPN patients confirmed that $32 \%$ have been seen or diagnosed with depression and $22.2 \%$ had received active treatment of mood disorder within the prior six months suggesting potential association in this population [40]. 
TABLE 3: Associations between MPNs and cytokines.

\begin{tabular}{|c|c|c|}
\hline $\begin{array}{l}\text { Inflammatory } \\
\text { marker* }^{*}\end{array}$ & Impact & Disorder \\
\hline B2MICG & Symptoms & MF \\
\hline BMP1 & Disease advancement & PMF \\
\hline BMP6 & Disease advancement & PMF \\
\hline BMP7 & Disease advancement & PMF \\
\hline BMP-Rcp2 & Disease advancement & PMF \\
\hline CD40L & Loss of appetite & MF \\
\hline CRP & Thrombosis; atherogenesis & PV, ET \\
\hline Ferritin & Pruritus & MF \\
\hline FGF & Marrow fibrosis & PV, ET, PMF \\
\hline HGF & Splenomegaly & $\mathrm{PMF}$ \\
\hline IFN & $\begin{array}{l}\text { Associated with } \\
\text { JAK2V617F }\end{array}$ & MF \\
\hline IL-12 & $\begin{array}{l}\text { Inferior survival; } \\
\text { transfusion requirements, } \\
\text { vascular complications }\end{array}$ & MF \\
\hline IL-15 & Inferior survival & MF \\
\hline IL-17A & $\begin{array}{l}\text { Associated with } \\
\text { JAK2V617F }\end{array}$ & MF \\
\hline IL-1B & $\begin{array}{l}\text { Associated with } \\
\text { JAK2V617F }\end{array}$ & MF \\
\hline IL-1RA & Splenomegaly & PMF \\
\hline IL-2R & $\begin{array}{l}\text { Inferior survival; } \\
\text { transfusion requirements }\end{array}$ & MF \\
\hline IL-8 & $\begin{array}{l}\text { Elevated blasts; } \\
\text { constitutional symptoms }\end{array}$ & MF \\
\hline IL-8 & $\begin{array}{l}\text { Associated with } \\
\text { JAK2V617F }\end{array}$ & MF \\
\hline IP-10 & Inferior survival & MF \\
\hline LEPTIN & Symptoms; weight loss & MF \\
\hline MIG & Splenomegaly & PMF \\
\hline PAL1 & Insomnia & MF \\
\hline PTX & Thrombosis; atherogenesis & PV, ET \\
\hline RANTES & Insomnia & MF \\
\hline TIMP1 & Symptoms & MF \\
\hline TNF-1 & Clonal expansion & $\begin{array}{l}\text { JAK2V617F+ } \\
\text { MPNs }\end{array}$ \\
\hline TNFRII & Symptoms & MF \\
\hline VCAM1 & Symptoms & MF \\
\hline VEGFb & Marrow fibrosis & PV, ET, PMF \\
\hline
\end{tabular}

ET: essential thrombocythemia; MF: myelofibrosis; MPN: myeloproliferative neoplasm; PMF: primary myelofibrosis; PV: primary myelofibrosis.

${ }^{*}$ Refer to Table 2 for definition.

The combination of cancer-related depression and fatigue also contributes to a sedentary lifestyle which further encourages a proinflammatory state that propagates symptoms. Multiple randomized controlled trials have demonstrated that cancer-related fatigue may be reduced through aerobic physical activity, potentially through modulation of cytokine production [37]. The mechanism has yet to be elucidated as intense physical activity has been shown to increase circulating levels of IL- 6 which subsequently stimulates the production of other anti-inflammatory cytokines including IL-1RA and IL-10, and inhibit proinflammatory cytokines such as TNF- $\alpha$. The MPN Fatigue Project is an international effort performed in collaboration with the MPN Forum aiming at evaluating the breadth and efficacy of current strategies targeting MPN fatigue [41]. The study remains ongoing and includes evaluation of treatments such as sleep deprivation, dietary supplements, and exercise.

4.2. Splenomegaly. Abdominal-related complaints are common among MPN patients, largely attributable to splenomegaly, portal hypertension, mechanical obstruction, and splenic infarcts. An independent source of morbidity and mortality complaints related to the abdomen has included early satiety $(76 \%)$, abdominal pain (63\%), abdominal discomfort (72\%), and weight loss (48\%) [3]. However, cytokines may also play an important role in the development of this symptom. Splenomegaly has been associated with expansion of the malignant clone from the bone marrow microenvironment to extramedullary sites including the spleen. TNF- $\alpha$, in particular, promotes clonal expansion in JAK2V617F positive MPNs [42, 43]. The development of splenomegaly has also been associated with specific cytokines including MIG, HGF, and IL-1RA [23]. However, the mechanism involved in stimulation has yet to be established. Interestingly, JAK2/STAT3 signaling has been shown to promote fibrosis, angiogenesis, and inflammation in the setting of portal hypertension, independent of the presence of malignancy suggesting that regional inflammation also plays a role in abdominal pain, whether or not cancer is present [44]. Thrombosis may result in a variety of abdominal complaints, particularly with ET and PV. An evaluation of 244 consecutive PV and ET patients demonstrated that patients within the highest CRP protein tertile had the highest rate of major thrombotic events [28]. Similarly patients demonstrating the lowest pentraxin 3 levels had higher risks for major thrombotic events. Of interest, values of hs-CRP and PTX3 also correlated with JAK2V617F allele burden.

Abdominal pain may also be exacerbated by cytokineinduced nerve hyperstimulation, both peripherally and centrally. Animal studies have shown increased expression of TNF- $\alpha$, IL-1, and IL- 6 after nerve injury, cytokines all disproportionately high in the MPN population. In addition, inflammation and trauma have been shown to induce peripheral nerve cell release of inflammatory cytokines within the central nervous system via glial stimulation [45]. The effects of cytokine-mediated pain were demonstrated in a recent study of patients with painful neuropathies where it was observed that this population had twofold higher levels of IL-2 mRNA and TNF- $\alpha$ mRNA in comparison to healthy controls [46]. The direct impact these cytokines have on nerves within MPN populations has yet to be investigated.

4.3. Microvascular, Cognitive Symptoms and Pruritus. Microvascular complaints typically refer to those symptoms that result from disease activity occurring at a capillary level. In the MPNs, these symptoms may include headaches, 
concentration problems, lightheadedness, dizziness, vertigo, numbness/tingling, and sexual dysfunction. Historically, neurocognitive disturbances have been attributed to cellular stasis and microthrombosis. Proinflammatory cytokine production is believed to be a contributor to cognitive impairment in cancer patients via the disruption of neurohormonal signaling and impaired creation of neurotransmitters. These neurotransmitters include serotonin, dopamine, and norepinephrine, all of which are critical to functions involving homeostasis of sleep, mood, and memory [47-49]. A recent analysis of patients treated with ruxolitinib in the COMFORT-II trials identified RANTES and Pall levels to correlate with the complaints of insomnia [50].

The role of inflammation in cognitive impairment has been intensely studied in both animal and human models. In an evaluation of IL-6 deficient animals, injection of lipopolysaccharide (LPS, shown to inhibit memory and learning in animals) failed to induce cognitive impairments suggesting IL-6 plays a key role in interrupting the process of memory and learning [49]. In human studies, elevated levels of IL-1, TNF- $\alpha$, IL-6, and CRP were also linked to impaired memory and neurodegenerative disorders in the elderly [5153]. Within hematological disorders, patients with elevated levels of IL- 6 were found to have worsened executive function [36]. Interestingly, AML and MDS patients with higher levels of IL- 8 were found to have improved memory. Pruritus has also been linked to the inflammatory cascade. A recent study of JAK2V617F transgenic mice demonstrated increased number of mast cells in those with the PV phenotype [54]. These mast cells represent a key source of prostaglandin, leukotriene, histamine, and tryptase, mediators of the inflammatory response with recognized ties to pruritus. A study evaluating symptoms of ruxolitinib treated patients within the COMFORT-II trial determined that baseline pruritus was associated with lower ferritin levels, a surrogate marker of inflammation [50]. Pruritus, most prominent in PV patients (65\%), may be tied to an inflammatory cellular response as well. Basophils have been instigated as a primary mediator for symptom development and studies have demonstrated that the number of constitutively activated and hypersensitive circulating basophils is increased in PV, correlating with the degree of pruritus [55]. In addition, mast cells may play a role. Recent studies using infrared thermography have documented mast cell degranulation due to temperature shifts with the release of pyrogenic factors such as interleukins, histamine, and leukotrienes [56, 57].

4.4. Constitutional Symptoms. Fevers, night sweats, and weight loss are foundational symptoms in MPNs. Both fevers and night sweats are recognized to be partially cytokine driven, typically by IL-1, IL-2, IL-6, TNF- $\alpha$, and IFN. Within other malignancies, IL- 6 has been found to correlate with the presence of B symptoms which serve as a prognostic factor in Chronic Lymphocytic Leukemia (CLL) and Hodgkin's lymphoma [58-60].

The development of MPN-associated weight loss is complex and relates to a variety of factors including splenomegaly, portal hypertension, and cancer-cachexia. The role of a cytokine-driven proinflammatory state as the nidus for cancer-cachexia is well supported by literature $[61,62]$. Defined as a process of dysregulated carbohydrate and fat metabolism with ongoing skeletal muscle breakdown, the presence of cancer-cachexia is linked to a dismal survival rate in comparison to cancer patients lacking this feature [63]. In cancer patients, TNF- $\alpha$ has been shown to induce proteolysis of skeletal muscle and furthermore enhance the expression of genes related to enzymes in the ubiquitindependent proteolytic pathway [64]. In a review of cytokine levels present in patients from the COMFORT-II trial, weight loss was associated with lower leptin levels and high CD40L was associated with loss of appetite. Whether these aberrant cytokines function to support cancer-cachexia or involve an alternative mechanism of action has yet to be investigated.

\section{Improving Symptoms by Targeting Cytokines}

Recognizing the substantial impact chronic inflammation has on the MPN symptom burden, attentions have turned to therapies demonstrating efficacy in reducing cytokines. As in other malignancies, improving physical activity and reducing fat intake may reduce inflammatory cytokines and improve survival [65]. In noncancer patients, increased physical activity has been shown to reduce TLR4 signaling and truncate release of inflammatory cytokines [66]. In obese subjects, proinflammatory cytokines have also been shown to be released by white fat which may be subsequently removed through physical activity. In addition to evaluating the effects of sedentary living on the MPN symptom burden, the final phases of the MPN Fatigue Project involve the development of comprehensive patient activity programs which may have subsequent impact on cytokine-induced symptomatology [41].

Recognizing the role constitutive Janus kinase signaling plays in inflammation, JAK2 inhibition has become a rational target for preventing cytokine dysregulation. A recent study evaluated meaningful changes in cytokine expression following 24 months of ruxolitinib therapy in 63 high-risk MF patients [67]. Ruxolitinib was able to induce profound reductions in the expression of TNF- $\alpha$ and MIP- $1 \alpha$ at both 4 weeks and 24 months. The expression of $\operatorname{IgE}$ was also strongly reduced in almost all patients with direct impact on the amount of activated anti-inflammatory macrophages. A similar study utilized the Functional Assessment of Cancer Therapy-Lymphoma (FACT-Lym) to evaluate symptoms of ruxolitinib treated patients within the COMFORT II trial and compare them to changes in cytokine levels [50]. Ten symptoms including fever, weight loss, fatigue, loss of appetite, pain, itching, sleeping well, lack of energy, night sweats, and trouble sleeping were assessed at baseline and weeks 8, 24, and 48. Treatment with ruxolitinib led to improved items of itching, night sweats, and weight loss with subsequent reduction in numerous cytokines. Loss of appetite improved over time and negatively correlated with decreases in IL-1RA levels in ruxolitinib treated patients.

The impact emerging JAK2 inhibitors such as momelotinib and pacritinib will have on cytokines is of high interest 
as they progress through clinical trials. Importantly, their limited hematological toxicities may be reflective of their selective inhibition of kinases. A recent investigation of pacritinib demonstrated that it selectively spares $J A K 1$ while inhibiting JAK2, JAK2V617F, FLT3, and IRAK1, an IL-1 receptor kinase associated with the inflammatory response and suppression of normal hematopoiesis [68]. Whether inhibition of IRAK1 is of clinical significance from a symptomatic standpoint has yet to be investigated. Other cellular signaling networks such as the PI3K-Akt-mTOR pathway impact cytokine development and represent novel targets for intervention. Similarly, as antifibrosing agents, hedgehog inhibitors, hypomethylating agents, histone deacetylation inhibitors, and HSP90 inhibitors enter the treatment landscape, knowledge of their impact on inflammation is of great interest.

\section{Conclusion}

Clear relationships exist between MPN symptoms and markers of inflammation. Though most data remains in early stages of investigation, knowledge gleaned from other malignancies has offered us potential mechanisms that explain these observed cytokine-symptom associations. A variety of MPN symptoms correlate with the presence of specific markers of inflammation. However, how these markers differ between MPN subtypes, change with disease progression, and relate to transformation remain unknown. The expanded access to targeted agents has provided a platform by which cytokine signals may be inhibited early within the cascade, limiting their potential for toxicity. It is with great anticipation that we venture into this uncharted territory of cytokinesymptom associations and explore novel therapies hosting high potential for symptomatic benefit.

\section{Conflict of Interests}

Geyer, Scherber, and Dueck have no conflict of interests to declare. Mesa works in consultancy for Novartis and in research for Incyte, Gilead, CTI, Genentech, Promedior, and NS Pharma.

\section{References}

[1] F. Cervantes, B. Dupriez, A. Pereira et al., "New prognostic scoring system for primary myelofibrosis based on a study of the International working group for myelofibrosis research and treatment," Blood, vol. 113, no. 13, pp. 2895-2901, 2009.

[2] N. Gangat, D. Caramazza, R. Vaidya et al., "DIPSS plus: a refined dynamic international prognostic scoring system for primary myelofibrosis that incorporates prognostic information from karyotype, platelet count, and transfusion status," Journal of Clinical Oncology, vol. 29, no. 4, pp. 392-397, 2011.

[3] R. Scherber, A. C. Dueck, P. Johansson et al., "The Myeloproliferative Neoplasm Symptom Assessment Form (MPN-SAF): international prospective validation and reliability trial in 402 patients," Blood, vol. 118, no. 2, pp. 401-408, 2011.

[4] R. Mesa, C. B. Miller, M. Thyne et al., "Impact of myeloproliferative neoplasms (MPNs) on patients' overall health and productivity: results from the MPN LANDMARK SURVEY in the United States," Blood, vol. 124, no. 21, p. 3183, 2014.

[5] H. Geyer, A. C. Dueck, R. M. Emanuel et al., "Insomnia, quality of life and MPN symptom burden: an analysis by the MPN quality of life international study group (MPN-QOL ISG)," Blood, vol. 122, no. 21, p. 4087, 2013.

[6] H. Geyer, A. C. Dueck, R. M. Emanuel et al., "Sexuality challenges, intimacy, and MPN symptom burden: an analysis by the MPN quality of life international study group (MPN-QOL ISG)," Blood, vol. 122, p. 4088, 2013.

[7] H. L. Geyer, R. M. Scherber, A. C. Dueck et al., "Distinct clustering of symptomatic burden among myeloproliferative neoplasm patients: retrospective assessment in 1470 patients," Blood, vol. 123, no. 24, pp. 3803-3810, 2014.

[8] R. A. Mesa, J. Niblack, M. Wadleigh et al., "The burden of fatigue and quality of life in myeloproliferative disorders (MPDs): an international Internet-based survey of 1179 MPD patients," Cancer, vol. 109, no. 1, pp. 68-76, 2007.

[9] R. A. Mesa, S. Schwager, D. Radia et al., "The Myelofibrosis Symptom Assessment Form (MFSAF): an evidence-based brief inventory to measure quality of life and symptomatic response to treatment in myelofibrosis," Leukemia Research, vol. 33, no. 9, pp. 1199-1203, 2009.

[10] R. A. Mesa, H. Kantarjian, A. Tefferi et al., "Evaluating the serial use of the myelofibrosis symptom assessment form for measuring symptomatic improvement: performance in 87 myelofibrosis patients on JAK1 and JAK2 inhibitor (INCB018424) clinical trial," Cancer, vol. 117, no. 21, pp. 4869-4877, 2011.

[11] S. Verstovsek, R. A. Mesa, S. K. Rhoades et al., "Phase I study of the JAK2 V617F inhibitor, LY2784544, in patients with myelofibrosis (MF), polycythemia vera (PV), and essential thrombocythemia (ET)," Blood, vol. 118, pp. 1213-1214, 2011.

[12] S. Verstovsek, R. A. Mesa, J. Gotlib et al., "A double-blind, placebo-controlled trial of ruxolitinib for myelofibrosis," The New England Journal of Medicine, vol. 366, no. 9, pp. 799-807, 2012.

[13] C. L. Andersen, N. B. Mortensen, T. W. Klausen, H. Vestergaard, O. W. Bjerrum, and H. C. Hasselbalch, "A phase ii study of vorinostat (MK-0683) in patients with primary myelofibrosis and post-polycythemia vera myelofibrosis," Haematologica, vol. 99, no. 1, pp. e5-e7, 2014.

[14] R. M. Emanuel, A. C. Dueck, H. L. Geyer et al., "Myeloproliferative neoplasm (MPN) symptom assessment form total symptom score: prospective international assessment of an abbreviated symptom burden scoring system among patients with MPNs," Journal of Clinical Oncology, vol. 30, no. 33, pp. 4098-4103, 2012.

[15] R. L. Levine, M. Wadleigh, J. Cools et al., "Activating mutation in the tyrosine kinase JAK2 in polycythemia vera, essential thrombocythemia, and myeloid metaplasia with myelofibrosis," Cancer Cell, vol. 7, no. 4, pp. 387-397, 2005.

[16] A. L.-F. Mui, "The role of STATs in proliferation, differentiation, and apoptosis," Cellular and Molecular Life Sciences, vol. 55, no. 12, pp. 1547-1558, 1999.

[17] H. C. Hasselbalch, "Perspectives on chronic inflammation in essential thrombocythemia, polycythemia vera, and myelofibrosis: is chronic inflammation a trigger and driver of clonal evolution and development of accelerated atherosclerosis and second cancer?" Blood, vol. 119, no. 14, pp. 3219-3225, 2012.

[18] C. A. Dinarello, "Historical insights into cytokines," European Journal of Immunology, vol. 37, supplement 1, pp. S34-S45, 2007.

[19] M. E. Figueroa, O. Abdel-Wahab, C. Lu et al., "Leukemic IDH1 and IDH2 mutations result in a hypermethylation phenotype, 
disrupt TET2 function, and impair hematopoietic differentiation," Cancer Cell, vol. 18, no. 6, pp. 553-567, 2010.

[20] H. Frederiksen, D. K. Farkas, C. F. Christiansen, H. C. Hasselbalch, and H. T. Sørensen, "Chronic myeloproliferative neoplasms and subsequent cancer risk: a Danish population-based cohort study," Blood, vol. 118, no. 25, pp. 6515-6520, 2011.

[21] H. C. Hasselbalch, "Chronic inflammation as a promotor of mutagenesis in essential thrombocythemia, polycythemia vera and myelofibrosis. A human inflammation model for cancer development?" Leukemia Research, vol. 37, no. 2, pp. 214-220, 2013.

[22] H. C. Hasselbalch, "Idiopathic myelofibrosis. Clinical aspects and studies on extracellular matrix metabolism," Danish Medical Bulletin, vol. 40, no. 1, pp. 39-55, 1993.

[23] A. Tefferi, R. Vaidya, D. Caramazza, C. Finke, T. Lasho, and A. Pardanani, "Circulating interleukin (IL)-8, IL-2R, IL-12, and IL15 levels are independently prognostic in primary myelofibrosis: a comprehensive cytokine profiling study," Journal of Clinical Oncology, vol. 29, no. 10, pp. 1356-1363, 2011.

[24] A. C. Dueck, C. S. Cleeland, R. Dantzer et al., "Cytokine profile changes in 309 myelofibrosis patients: comparison of JAK1/ JAK2 inhibitor therapy vs. placebo-correlative analysis from the comfort-I trial," Blood, vol. 122, no. 21, p. 4074, 2013.

[25] R. Vaidya, N. Gangat, T. Jimma et al., "Plasma cytokines in polycythemia vera: phenotypic correlates, prognostic relevance, and comparison with myelofibrosis," American Journal of Hematology, vol. 87, no. 11, pp. 1003-1005, 2012.

[26] E. Pourcelot, C. Trocme, J. Mondet, S. Bailly, B. Toussaint, and P. Mossuz, "Cytokine profiles in polycythemia vera and essential thrombocythemia patients: clinical implications," Experimental Hematology, vol. 42, no. 5, pp. 360-368, 2014.

[27] L. Catani, L. Rossi, D. Sollazzo et al., "Crucial factors of the inflammatory microenvironment promote maintenance of the malignant hemopoietic clone of myelofibrosis by stimulating survival and inhibiting proliferation of $\mathrm{CD} 34^{+}$stem/progenitor cells," Blood, vol. 124, no. 21, p. 3220, 2014.

[28] T. Barbui, A. Carobbio, G. Finazzi et al., "Inflammation and thrombosis in essential thrombocythemia and polycythemia vera: different role of C-reactive protein and pentraxin 3," Haematologica, vol. 96, no. 2, pp. 315-318, 2011.

[29] S.-Y. Yoon, C.-Y. Li, R. V. Lloyd, and A. Tefferi, "Bone marrow histochemical studies of fibrogenic cytokines and their receptors in myelodysplastic syndrome with myelofibrosis and related disorders," International Journal of Hematology, vol. 72, no. 3, pp. 337-342, 2000.

[30] M. C. Le Bousse-Kerdilès and M. C. Martyré, "Dual implication of fibrogenic cytokines in the pathogenesis of fibrosis and myeloproliferation in myeloid metaplasia with myelofibrosis," Annals of Hematology, vol. 78, no. 10, pp. 437-444, 1999.

[31] O. Bock, J. Höftmann, K. Theophile et al., "Bone morphogenetic proteins are overexpressed in the bone marrow of primary myelofibrosis and are apparently induced by fibrogenic cytokines," American Journal of Pathology, vol. 172, no. 4, pp. 951-960, 2008.

[32] A. Tabarroki, H. Rogers, V. Visconte et al., "The molecular and cytokine profile of triple-negative (JAK2 V617F, JAK2 exon 12, MPL negative) myelofibrosis, a myeloproliferative neoplasm with distinct clinico-pathologic characteristics," Blood, vol. 633, p. 3805, 2012, ASH Annual Meeting.

[33] D. P. Lawrence, B. Kupelnick, K. Miller, D. Devine, and J. Lau, "Evidence report on the occurrence, assessment, and treatment of fatigue in cancer patients," Journal of the National Cancer Institute. Monographs, no. 32, pp. 40-50, 2004.

[34] J. E. Bower, P. A. Ganz, M. R. Irwin, L. Kwan, E. C. Breen, and S. W. Cole, "Inflammation and behavioral symptoms after breast cancer treatment: do fatigue, depression, and sleep disturbance share a common underlying mechanism?" Journal of Clinical Oncology, vol. 29, no. 26, pp. 3517-3522, 2011.

[35] R. Kurzrock, "The role of cytokines in cancer-related fatigue," Cancer, vol. 92, no. 6, pp. 1684-1688, 2001.

[36] C. A. Meyers, M. Albitar, and E. Estey, "Cognitive impairment, fatigue, and cytokine levels in patients with acute myelogenous leukemia or myelodysplastic syndrome," Cancer, vol. 104, no. 4, pp. 788-793, 2005.

[37] J. E. Bower, P. A. Ganz, N. Aziz, R. Olmstead, M. R. Irwin, and S. W. Cole, "Inflammatory responses to psychological stress in fatigued breast cancer survivors: relationship to glucocorticoids," Brain, Behavior, and Immunity, vol. 21, no. 3, pp. 251-258, 2007.

[38] J. E. Bower, P. A. Ganz, K. A. Desmond et al., "Fatigue in longterm breast carcinoma survivors: a longitudinal investigation," Cancer, vol. 106, no. 4, pp. 751-758, 2006.

[39] D. L. Musselman, A. H. Miller, M. R. Porter et al., "Higher than normal plasma interleukin- 6 concentrations in cancer patients with depression: preliminary findings," American Journal of Psychiatry, vol. 158, no. 8, pp. 1252-1257, 2001.

[40] R. M. Scherber, Z. Senyak, A. C. Dueck et al., "High prevalence of mood disorders in MPNs and their possible role in MPN related fatigue," Blood, vol. 124, no. 21, p. 3173, 2014.

[41] R. M. Scherber, Z. Senyak, A. C. Dueck et al., "The impact of medical comorbidities in MPN-related fatigue," Blood, vol. 124, p. 1862, 2014.

[42] J. Li, D. P. Sejas, X. Zhang et al., "TNF- $\alpha$ induces leukemic clonal evolution ex vivo in Fanconi anemia group C murine stem cells," Journal of Clinical Investigation, vol. 117, no. 11, pp. 3283-3295, 2007.

[43] A. G. Fleischman, K. J. Aichberger, S. B. Luty et al., "TNF $\alpha$ facilitates clonal expansion of JAK2V617F positive cells in myeloproliferative neoplasms," Blood, vol. 118, no. 24, pp. 63926398, 2011.

[44] D. Wang, J. Yin, R. Dong et al., "Inhibition of Janus kinase-2 signalling pathway ameliorates portal hypertensive syndrome in partial portal hypertensive and liver cirrhosis rats," Digestive and Liver Disease, vol. 47, pp. 315-323, 2015.

[45] L. R. Watkins and S. F. Maier, "The pain of being sick: implications of immune-to-brain communication for understanding pain," Annual Review of Psychology, vol. 51, pp. 29-57, 2000.

[46] N. Üçeyler, J. P. Rogausch, K. V. Toyka, and C. Sommer, "Differential expression of cytokines in painful and painless neuropathies," Neurology, vol. 69, no. 1, pp. 42-49, 2007.

[47] F. Shintani, S. Kanba, T. Nakaki et al., "Interleukin-1 $\beta$ augments release of norepinephrine, dopamine, and serotonin in the rat anterior hypothalamus," The Journal of Neuroscience, vol. 13, no. 8, pp. 3574-3581, 1993.

[48] P. S. Mohankumar, S. Thygarajan, and S. K. Quadri, "Interleukin-1 stimulates the release of dopamine and dihydroxyphenylacetic acid from the hypothalamus in vivo," Life Sciences, vol. 48, no. 9, pp. 925-930, 1991.

[49] N. L. Sparkman, J. B. Buchanan, J. R. R. Heyen, J. Chen, J. L. Beverly, and R. W. Johnson, "Interleukin-6 facilitates lipopolysaccharide-induced disruption in working memory and expression of other proinflammatory cytokines in hippocampal 
neuronal cell layers,' The Journal of Neuroscience, vol. 26, no. 42, pp. 10709-10716, 2006.

[50] C. N. Harrison, G. Barosi, A. M. Vannucchi et al., "The relationship between cytokine levels and symptoms in patients (Pts) with myelofibrosis (MF) from COMFORT-II, a phase 3 study of ruxolitinib (RUX) vs best available therapy (BAT)," Blood, vol. 122, no. 21, p. 4070, 2013.

[51] K. S. Krabbe, A. Reichenberg, R. Yirmiya, A. Smed, B. K. Pedersen, and H. Bruunsgaard, "Low-dose endotoxemia and human neuropsychological functions," Brain, Behavior, and Immunity, vol. 19, no. 5, pp. 453-460, 2005.

[52] K. S. Krabbe, M. Pedersen, and H. Bruunsgaard, "Inflammatory mediators in the elderly," Experimental Gerontology, vol. 39, no. 5, pp. 687-699, 2004.

[53] F. Zipp and O. Aktas, "The brain as a target of inflammation: common pathways link inflammatory and neurodegenerative diseases," Trends in Neurosciences, vol. 29, no. 9, pp. 518-527, 2006.

[54] X. Jin, W. Zhao, A. Kirabo et al., "Elevated levels of mast cells are involved in pruritus associated with polycythemia vera in JAK2V617F transgenic mice," Journal of Immunology, vol. 193, no. 2, pp. 477-484, 2014.

[55] L. Pieri, C. Bogani, P. Guglielmelli et al., "The JAK2V617 mutation induces constitutive activation and agonist hypersensitivity in basophils from patients with polycythemia vera," Haematologica, vol. 94, no. 11, pp. 1537-1545, 2009.

[56] J. Wang, T. Ishii, W. Zhang et al., "Involvement of mast cells by the malignant process in patients with Philadelphia chromosome negative myeloproliferative neoplasms," Leukemia, vol. 23, no. 9, pp. 1577-1586, 2009.

[57] N. Jackson, D. Burt, J. Crocker, and B. Boughton, "Skin mast cells in polycythaemia vera: relationship to the pathogenesis and treatment of pruritus," British Journal of Dermatology, vol. 116, no. 1, pp. 21-29, 1987.

[58] J. F. Seymour, M. Talpaz, F. Cabanillas, M. Wetzler, and R. Kurzrock, "Serum interleukin-6 levels correlate with prognosis in diffuse large-cell lymphoma," Journal of Clinical Oncology, vol. 13, no. 3, pp. 575-582, 1995.

[59] J. F. Seymour, M. Talpaz, F. B. Hagemeister, F. Cabanillas, and R. Kurzrock, "Clinical correlates of elevated serum levels of interleukin-6 in patients with untreated Hodgkin's disease," The American Journal of Medicine, vol. 102, no. 1, pp. 21-28, 1997.

[60] J. Cortes and R. Kurzrock, "Interleukin-10 in non-Hodgkin's lymphoma," Leukemia \& Lymphoma, vol. 26, no. 3-4, pp. 251259, 1997.

[61] E. J. B. Ramos, S. Suzuki, D. Marks, A. Inui, A. Asakawa, and M. M. Meguid, "Cancer anorexia-cachexia syndrome: cytokines and neuropeptides," Current Opinion in Clinical Nutrition and Metabolic Care, vol. 7, no. 4, pp. 427-434, 2004.

[62] J. M. Argilés, S. Busquets, and F. J. López-Soriano, "The pivotal role of cytokines in muscle wasting during cancer," International Journal of Biochemistry and Cell Biology, vol. 37, no. 10, pp. 20362046, 2005

[63] M. J. Tisdale, "Biology of cachexia," Journal of the National Cancer Institute, vol. 89, no. 23, pp. 1763-1773, 1997.

[64] M. Bossola, M. Muscaritoli, P. Costelli et al., "Increased muscle ubiquitin mrna levels in gastric cancer patients," American Journal of Physiology-Regulatory Integrative and Comparative Physiology, vol. 280, no. 5, pp. R1518-R1523, 2001.

[65] B. Seruga, H. Zhang, L. J. Bernstein, and I. F. Tannock, "Cytokines and their relationship to the symptoms and outcome of cancer," Nature Reviews Cancer, vol. 8, no. 11, pp. 887-899, 2008.
[66] M. G. Flynn and B. K. McFarlin, "Toll-like receptor 4: link to the anti-inflammatory effects of exercise?" Exercise and Sport Sciences Reviews, vol. 34, no. 4, pp. 176-181, 2006.

[67] H. M. Kvasnicka, J. Thiele, C. E. Bueso-Ramos et al., "Changes in activated bone marrow macrophages and mast cells in patients with myelofibrosis following ruxolitinib therapy," Blood, vol. 124, no. 21, p. 3184, 2014.

[68] J. Singer, S. Al-Fayoumi, H. Ma, R. S. Komrokji, R. Mesa, and S. Verstovsek, "Comprehensive kinase profile of pacritinib, a nonmyelosuppressive JAK2 kinase inhibitor in phase 3 development in primary and post ET/PV myelofibrosis," Blood, vol. 124, no. 21, p. 1874, 2014. 


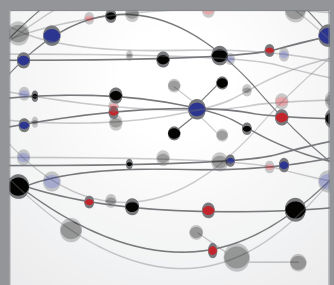

The Scientific World Journal
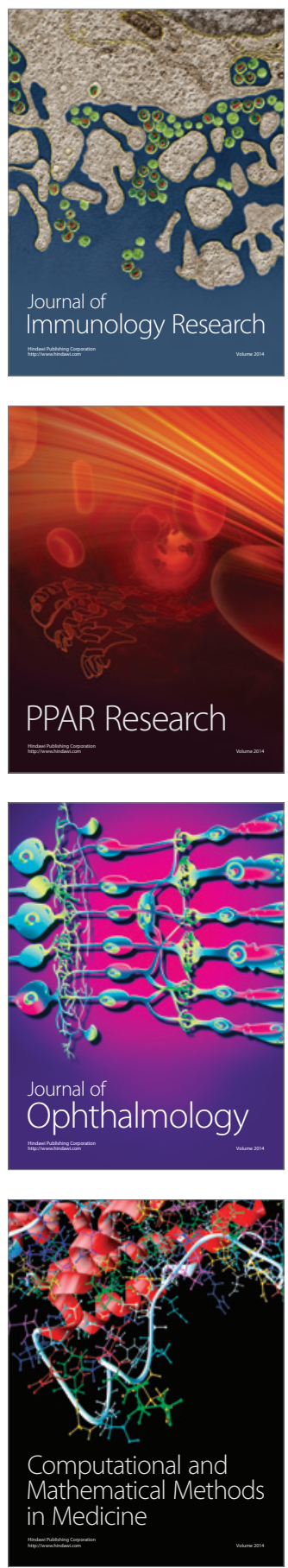

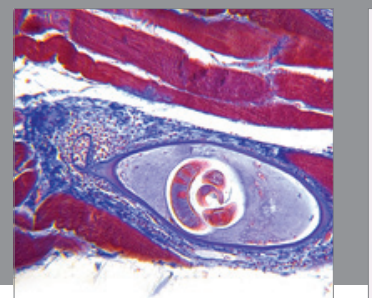

Gastroenterology

Research and Practice
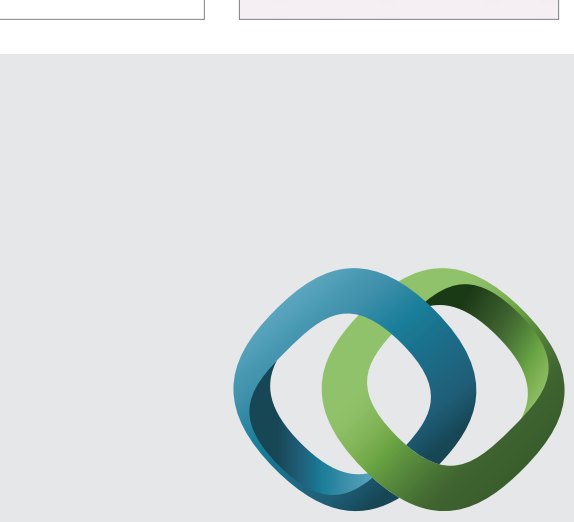

\section{Hindawi}

Submit your manuscripts at

http://www.hindawi.com
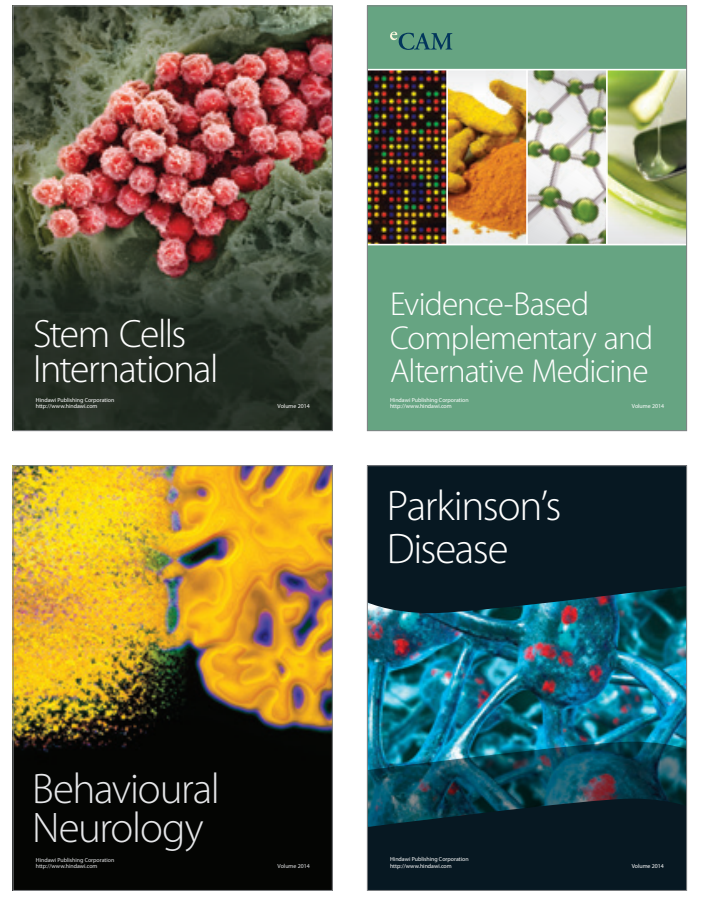
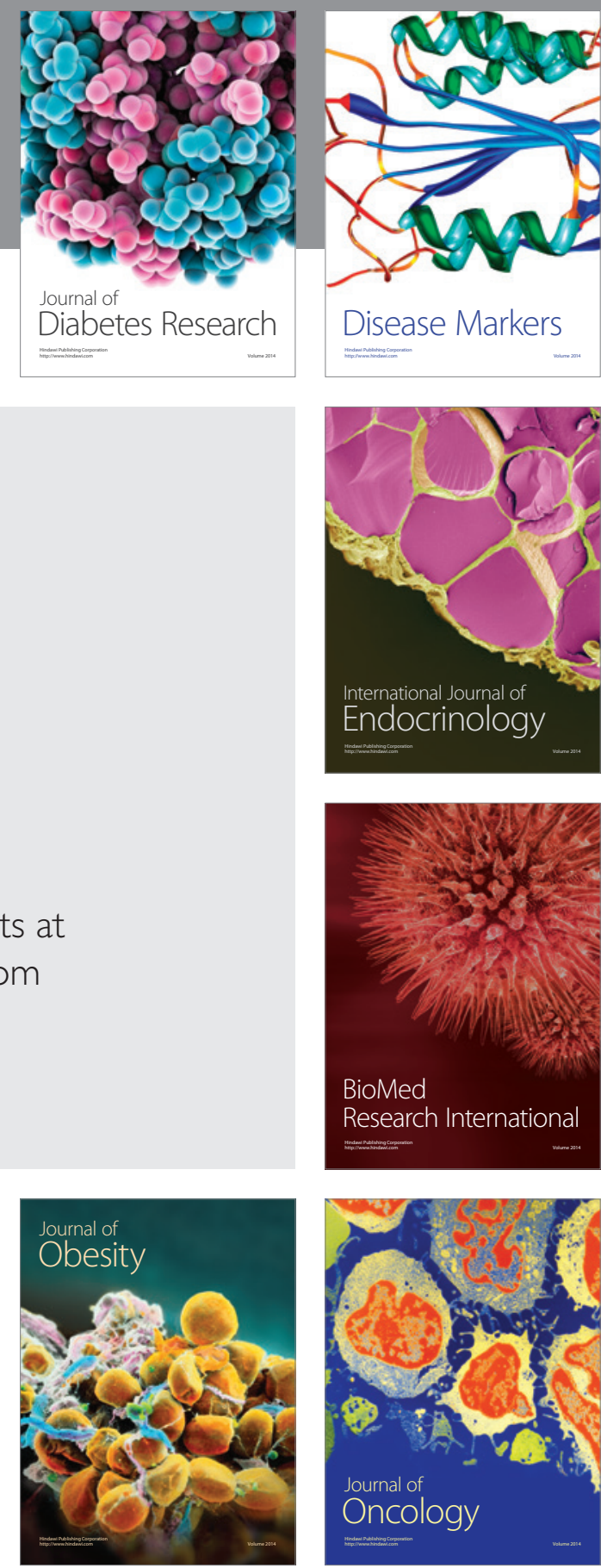

Disease Markers
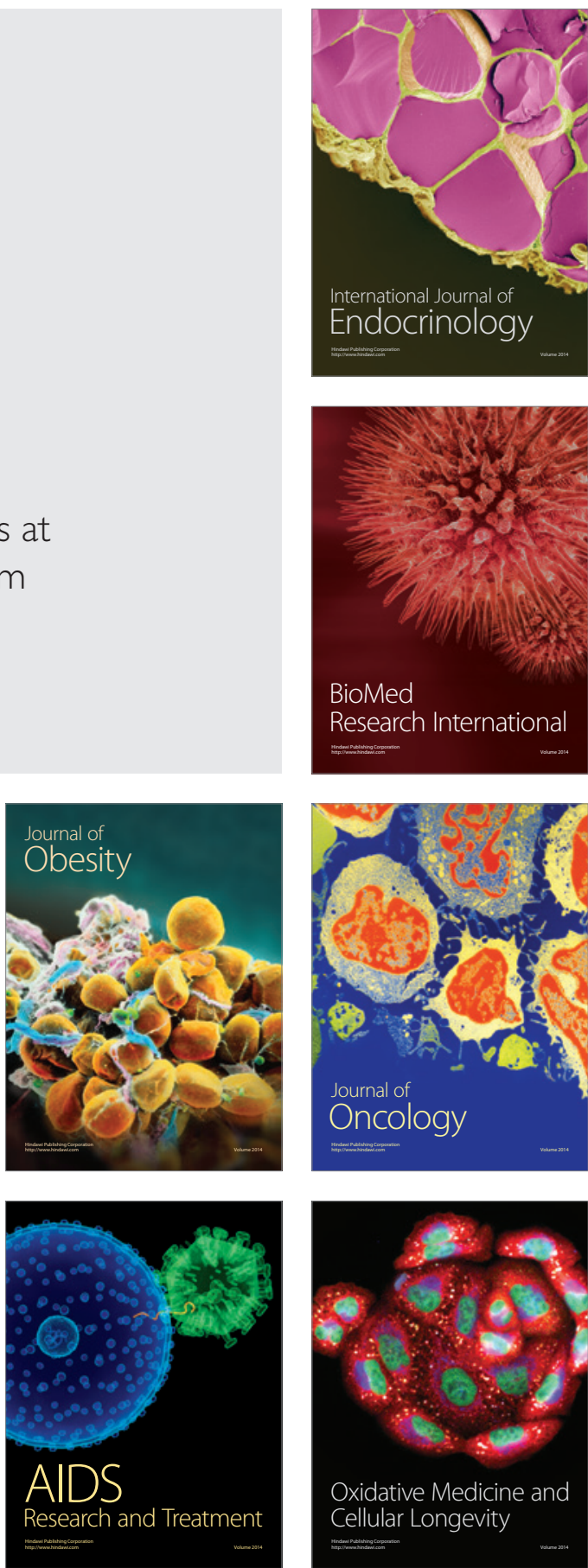